\title{
Developing a visual learning tool to aid the studying of antimicrobial spectrum of activity
}

\author{
Gagani Athauda ${ }^{1}$, Rebecca L Toonkel ${ }^{2}$, Sanaz B Kashan ${ }^{2}$ \\ ${ }^{I}$ Cellular Biology and Pharmacology, Herbert Wertheim College of Medicine, Florida International University, USA, \\ ${ }^{2}$ Department of Humanities, Health, and Society, Herbert Wertheim College of Medicine, Florida International \\ University, USA
}

Background: As a general principal in antimicrobial therapy, the goal is to administer an antibiotic with the narrowest spectrum of activity that would be capable of affecting a particular microorganism when the identity is known. But in many cases, patients are treated empirically with an antimicrobial which has a broad spectrum of activity until the laboratory results identify the pathogenic microorganism. Therefore it is fundamental to have the knowledge of antibiotics' spectrum of activity when treating infectious diseases. One of the main areas of difficulty for teaching and learning antimicrobial pharmacology is mastering the spectrum of activity of antimicrobial drugs. We aim to remedy this and strengthen the knowledge of antimicrobial pharmacology needed for the treatment of infectious diseases.

Methods: We have developed a visual tool to aid in mastering the spectrum of antimicrobial drugs. This tool focuses on most commonly used antimicrobials drugs and/or antimicrobials used for the treatment of serious infections. It uses color bars to show the spectrum of activity of a drug that would affect different genres of microorganism. This visual tool is incorporated to a series of short recorded videos which serves as self-study preparatory material as well as to the large group case based discussions. Students' performance related to the antimicrobial spectrum of activity is assessed using inhouse and or NBME MCQ's during multiple courses in P1 and P2 and a grading rubric developed by the faculty in P3.

Results: Outcome measures of the intervention are assessed at P2-P3 interface (pre-clinical students about to enter clinical clerkships) and P4-residency interface (students about to graduate medical school). Assessments consist of USMLE style MCQ's to assess antimicrobial spectrum of activity and a short survey using a 5-point Likert scale to assess perception of confidence in the subject matter.

Conclusions: Developing a visual learning tool to aid the studying of antimicrobial spectrum of activity will equip the preclinical students entering the clinical clerkships and 4th year medical students entering residency training to build their confidence and knowledge of antimicrobial spectrum of activity as well as strengthen the knowledge for rational prescribing of antimicrobials in treating infectious diseases. 